\title{
Can Somatostatin Receptor Analogs Re-Differentiate Pancreatic Neuroendocrine Tumors: A Case Report
}

\author{
Nada Younes ${ }^{1 *}$, Anne-Sophie Azzi ${ }^{2}$, Bassam Abboud ${ }^{3}$, Chawki Atallah ${ }^{1}$ \\ ${ }^{1}$ Division of Endocrinology, Hotel-Dieu de France university hospital, Saint-Joseph University, Ashrafieh, \\ Lebanon \\ ${ }^{2}$ Division of Endocrinology, Lebanese Hospital Geitaoui, Ashrafieh, Lebanon \\ ${ }^{3}$ Division of general surgery, Hotel-Dieu de France university hospital, Saint-Joseph University, Ashrafieh, \\ Lebanon
}

*Corresponding Author: Nada Younes, Division of Endocrinology, Hotel-Dieu de France university hospital, Saint-Joseph University, Ashrafieh, Lebanon.

\begin{abstract}
Ectopic Cushing's syndrome due to a pancreatic neuroendocrine tumor ( $p$-NET) is uncommon. Available information regarding effective treatment of pancreatic neuroendocrine neoplasms is limited, especially in grade 3 (G3) NETs with features of carcinoma. A 41-year-old female presented with a newly discovered round puffy face, severe resistant epigastric pain, new onset hyperglycemia and uncontrolled hypertension. Imaging tests performed, and cytology analysis revealed a well-differentiated (WD) high-grade p-NET producing ACTH, indistinguishable from a G3 neuroendocrine carcinoma due to over expression of p53 and a normal expression of ATRX. Regression of pancreatic mass and decrease in her ACTH and cortisol levels were seen after somatostatin receptor analog (SSTRA) therapy and steroidogenesis inhibitors. She underwent a distal pancreatectomy and splenectomy following medical treatment and was found to be cured on followup imaging and lab results. The final pathology report showed tumor re-differentiation. Somatostatin receptor analogs are known to reduce tumor bulk. Nonetheless, to our knowledge, no downgrading effect has been previously described in the literature. We present a rare case of a WD high grade-p-NET mimicking a NEC that was re-differentiated following neoadjuvant treatment with SSTRA.
\end{abstract}

Keywords: neuroendocrine tumor, Cushing syndrome, somatostatin, re-differentiation.

\section{INTRODUCTION}

Adrenocorticotropin (ACTH) - producing pancreatic neuroendocrine tumors (p-NETs), leading to Cushing's syndrome (CS) are rare and are responsible for $4-16 \%$ of all ectopic CS (1). They tend to be large, metastatic tumors with a malignant behavior (2). In the 2017 revised WHO classification for $\mathrm{p}$-NETs, grade 3 (G3) NET was introduced as a novel category. Its diagnosis remains however challenging and subject to many pitfalls especially when distinguishing it from G3 neuroendocrine carcinomas (NECs). Results from the CLARINET study (3) showed improvement of progression free survival in grades 1 and 2 NETs, with somatostatin receptor analogs (SSTRA), due to their antiproliferative effects (4). As for G3 NETs, treatment modalities are still poorly outlined. Here, we present a rare functioning high-grade p-NET with both well differentiated (WD) features and carcinoma features detected by ${ }^{68} \mathrm{Ga}$-DOTATATE and ${ }^{18} \mathrm{~F}$ FDG positron emission tomography-computed tomography (PET/CT). When treated preoperatively with SSTRA, both volume reduction and downgrading of the tumor were achieved.

\section{Case Presentation}

A 41-year-old female was admitted for a twoweek history of nausea and intense epigastric pain unresponsive to analgesics. On physical examination, she had a round puffy face, hypertension and a newly diagnosed hyperglycemia with a fasting blood glucose of $211 \mathrm{mg} / \mathrm{dL}$. During her hospital stay, she developed severe hypokalemia (as low as 2.6 $\mathrm{mmol} / \mathrm{L}$ ) requiring IV and oral potassium supplementation and treatment with spironolactone $25 \mathrm{mg} 1$ tablet TID.A computed tomography (CT) scan of abdomen and pelvis revealed a $67-\mathrm{mm}$ lobulated heterogeneous mass 
of the tail of the pancreas, obstructing the splenic vein, with collateral circulation and infiltration of the splenic artery and multiple retroperitoneal sub-centimetric lymph nodes (LNs). Subsequently, an endoscopic ultrasound confirmed a 35-mm caudal pancreatic lesion. Fine needle aspiration (FNA) showed a morphologically WD grade 3 (G3) (ki-67 index $27 \%$ ) neuroendocrine proliferation and no nonneuroendocrine components (Figure 1).
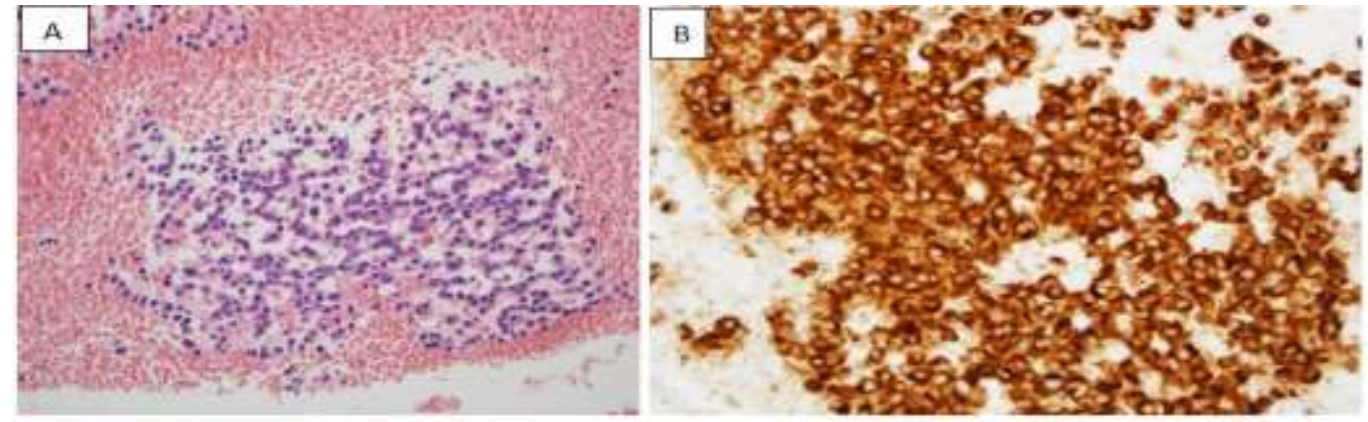

Figure1. Cytological images of the tumor. A (H\&E stain, x100) demonstrates one-dimensional trabecular pattern of small to medium sized cells with round nuclei and derepressed chromatin. Cell molding can also be seen. B (synaptophysin staining, x400) shows an intense diffuse positive staining to synaptophysin, a neuroendocrine marker.

Overexpression of $\mathrm{p} 53$ and absence of alpha-thalassemia/mental retardation X-linked (ATRX) mutation were in favor of a neuroendocrine carcinoma (NEC). Unfortunately, ACTH staining of tumor cells was not available in our hospital. Magnetic resonance imaging (MRI) of the liver showed multiple suspicious millimetric nodules. Both a ${ }^{68}$ Gallium DOTATATE PET-CT scan and a ${ }^{18} \mathrm{~F}$ FDG PET-CT showed a $7.5-\mathrm{cm}$ tumor of the tail of the pancreas with abnormal uptake (respectively, SUV $\max =50$ and SUV $\max =7$ ), without liver metastases (LM). Cancer antigen 19-9 (Ca 19-9) and carcinoembryonic antigen (CEA) were normal.

Laboratory tests confirmed a CS: a nonsuppressed morning plasma cortisol after an overnight low-dose dexamethasone suppression test $(1040 \mathrm{ng} / \mathrm{mL}$, suppressed values when $<18$ $\mathrm{ng} / \mathrm{mL}$ ), a midnight plasma cortisol of 1080 $\mathrm{ng} / \mathrm{mL}$ (normal values $<50 \mathrm{ng} / \mathrm{mL}$ ) and a nonsuppressed cortisol of $1250 \mathrm{ng} / \mathrm{mL}$ (suppressed values when $<18 \mathrm{ng} / \mathrm{mL}$ ) 6 hours after the last dose of dexamethasone following a two-day low-dose suppression test $(0.5 \mathrm{mg}$ q6hrs for 48hrs). Her urinary free cortisol was $2467 \mathrm{mcg} / 24 \mathrm{~h}$ (normal $<45 \mathrm{mcg} / 24 \mathrm{~h}$ ). ACTH dependent CS was confirmed after a morning ACTH of $342 \mathrm{pg} / \mathrm{mL}$ and midnight ACTH of $265 \mathrm{pg} / \mathrm{mL}$ (normal values respectively, 10-50 $\mathrm{pg} / \mathrm{mL}$ and $<5-10 \mathrm{pg} / \mathrm{mL}$ ). A pituitary MRI revealed no adenoma. She was diagnosed with an ectopic CS due to an ACTH producing high grade $\mathrm{p}-\mathrm{NET}$.
She was treated with steroidogenesis inhibitors (SIs) (ketoconazole $200 \mathrm{mg}$ TID and metyrapone $250 \mathrm{mg} 4$ times daily) and 100 micrograms of octreotide subcutaneously every 8 hours, which was then decreased to 100 micrograms every 12 hours following biochemical improvement: morning cortisol and ACTH decreased to $44.4 \mathrm{ng} / \mathrm{mL}$ (normal 100$200 \mathrm{ng} / \mathrm{mL}$ ) and $182 \mathrm{pg} / \mathrm{mL}$ (normal 10-50 $\mathrm{pg} / \mathrm{mL}$ ), respectively. She was discharged on octreotide LAR $30 \mathrm{mg}$ once a month, metyrapone and ketoconazole. A month later, her ACTH level normalized to $46.4 \mathrm{pg} / \mathrm{mL}$, and progressively declined to $17.6 \mathrm{pg} / \mathrm{mL}$ at her 3 month follow up, with a relatively low morning cortisol of $58.6 \mathrm{ng} / \mathrm{mL}$. A decrease of $16 \%$ in the volume of the pancreatic mass was noted on repeat abdominal MRI and the suspicious liver nodules were in fact compatible with biliary hamartomas. She was given glucocorticoids as per the block and treat regimen and subsequently underwent a distal pancreatectomy and splenectomy. The final pathology report noted a $7 \mathrm{~cm}$ grade $2(\mathrm{G} 2)(\mathrm{Ki} 67=15 \%)$ NET, with peripancreatic fat infiltration, neoplastic thrombosis of splenic vein, parietal infiltration of splenic artery and 2 peripancreatic metastatic LN. There were no G3 regions within the specimen. Tumor tissue stained positively and diffusely to pancytokeratine, chromogranin A and synaptophysin. P53 was not overexpressed while ATRX mutation as positive. Three months postoperatively, there was no residual disease on CT scan and her ACTH and cortisol levels, were normal; respectively $15.1 \mathrm{pg} / \mathrm{mL}$ and 119 $\mathrm{ng} / \mathrm{mL}$, while maintaining therapy with octreotide LAR $30 \mathrm{mg}$ monthly. 


\section{DISCUSSION}

Our patient presented with a WD ACTHsecreting p-NET. Based on initial cytology report, she had a high-grade tumor but exhibited molecular features of carcinoma: normal expression of ATRX and overexpression of $\mathrm{p} 53$. In the 2017 revised WHO classification, a new category of WD NETs was described almost exclusively in the pancreas (5) with a mitotic count $>20 / 10$ high power fields (HPFs) and/or ki-67 proliferative index $>20 \%$ corresponding to a G3. Nonetheless, distinction between G3 NETs and G3 NECs is not always easy. Contrary to p-NECs, differentiated G3 tumors conserve chromogranin and SST receptors (SSTRs) expression and can produce functional hormones (6). They are characterized by positive staining for neuroendocrine markers, chromogranin A and synaptophysin (6) and a coarse chromatin with a 'salt-and-pepper' appearance $(5,6)$. In cases of a difficult morphologic diagnosis, the 2017 WHO classification authors proposed the adjunct use of molecular markers detected by immunohistochemistry (IHC). These included p53 mutations and retinoblastoma $(\mathrm{Rb})$ protein loss, almost exclusively seen in PD-NECs $(5,7)$ and loss of ATRX and death domain-associated (DAXX) proteins expression in up to $40 \%$ of WD-p-NETs $(5,7)$. Therefore, tumors are classified as G3 NET when the phenotype is $\mathrm{p} 53-\mathrm{Rb}+\mathrm{DAXX} \pm \mathrm{ATRX} \pm$ or G3 NEC when $\mathrm{p} 53+\mathrm{Rb}-\mathrm{DAXX}+\mathrm{ATRX}+$ (8). Concordance between cytology specimen from FNAs and the final pathology report obtained from surgical specimen in neuroendocrine tumors, has been documented in up to $89.5 \%$ of cases (9).

In addition, the tumor was detected on both ${ }^{68}$ Ga-DOTATATE PET-CT and ${ }^{18}$ FDG-PETCT, demonstrating the presence of SSTRs as well as a high proliferative rate. This further confirmed the initial cytology report suggesting features of both WD NET and NEC. 70\% of pNETs express SSTRs, especially subtype 2, and can be detected using somatostatin analogs functional imaging $(2,7)$. Somatostatin receptor-based $68 \mathrm{Ga}$-PET-CT imaging (68 GaDOTATATE or 68 Ga-DOTANOC) is recommended for diagnosis and follow up of NETs because of its superiority to conventional imaging and octreoscan (10). When SSTR expression by NETs is straightforward either on octreoscan or by IHC, SSTRA are indicated because of their anti-hormonal and antiproliferative effects (7). Functional isotopic imaging may also play a role in determining prognosis of p-NETs: it is referred to as the metabolic grade (10). In fact, when tumors show uptake on SST analogs scintigraphy or PET/CT, they are more likely to be WD, are negative on FDG PET and have a better clinical outcome (10). Conversely, when tumors are avid on FDG-PET scans, even for grade 1 (G1) tumors, the intensity of uptake is associated with a worse outcome and increased tumor progression $(2,10)$. They are usually PD-NECs and G3 NETs, rarely avid on octreoscans $(2,10)$. Based on the stated clinical, cytology and imaging features, we decided to initiate treatment with a SSTRA to reduce tumor burden before surgery. Indeed, when tumor is bulky, a neoadjuvant approach can be applied, using either SSTRA and/or interferon alpha (INF $\alpha)$ or radionuclide therapy, to facilitate surgical resection $(2,7,10)$. Surprisingly, our patient's tumor regressed by more than $15 \%$ preoperatively. Results from the CLARINET (3) and PROMID (11) studies clearly demonstrate tumor shrinkage in response to SSTRA, particularly in low or intermediate grade tumors. The ideal candidates would be patients with a low ki-67 index and a low hepatic burden on MRI (7). Moreover, we not only demonstrated a reduction in tumor volume, but also a downgrading of tumor on final pathology following resection. Antiproliferative actions of SSTRA have been extensively documented, yet it is possible that they may have re-differentiation capacity, perhaps downgrading and re-differentiating the tumor, similar to thyroid cancer re differentiation: from a G3 NEC to a G2 NET, a hypothesis that warrants further study. This was previously described in a case report with cytotoxic chemotherapy but not in SSTRAs (12).

Moreover, the decision to surgically remove the tumor was undertaken in the absence of documented metastases on imaging. Curative surgery in the absence of hepatic or extra hepatic disease is the recommended course of action for a NET (13). However, if multiple inoperable hepatic or extra hepatic diseases are present, systemic therapy is indicated (13): biotherapy with SSTRA and/or INF $\alpha$ in cases of WD functional NETs. Seeing that G3 NET is a relatively new category, clear recommendations are yet to be established. The most important negative predictors of survival in patients on SSTRA are a high ki-67 proliferative index and increased hepatic tumor burden on MRI (7). 
Negative predictors of survival in patients with CS due to NETs are severity of hypercortisolemia ( $>3$-fold increase in serum cortisol or $>5$-fold increase in $24 \mathrm{~h}$ urine free cortisol and ACTH), the presence of hypokalemia, diabetes mellitus and distant metastases (14), most of which were present in our patient. The positive response seen in our patient is rather unusual. Another potential effect, recently proposed, is that of biguanides on inhibiting tumor progression (15).Finally, it is also possible that octreotide therapy may have had an added benefit to the treatment of CS as both ACTH and cortisol levels decreased. However, we are unable to quantify this effect, especially that the patient was treated simultaneously with ketoconazole and metyrapone as is recommended for a rapid clinical improvement (16). Few case reports described a positive response to therapy targeting the tumor producing ectopic ACTH rather than the hypercortisolemia itself $(17,18)$, using either a SSTRA or dopamine agonists. The effects of SSTRA were most noticeable when combined with a glucocorticoid receptor antagonist to increase expression of SSTR subtype 2 (16).

\section{CONCLUSION}

We described a rare case of an ACTHproducing WD-high-grade-p-NET with features of carcinoma on cytology and an aggressive clinical behavior, responding nonetheless to neoadjuvant treatment with SSTRA and SIs and curative surgery. Our findings shed light on a potential new role for SSTRA in NETs therapy. Perhaps, future studies using functional imaging will demonstrate a re-differentiating effect of SSTRA leading to downgrading of NETs, such as is described in thyroid cancers.

\section{Patient Consent}

Informed consent has been obtained from the patient for publication of the case report and accompanying images.

\section{DISCLOSURE SUMMARY}

This research did not receive any specific grant from any funding agency in the public, commercial or not-for-profit sector. All authors declare no conflicts of interest. All authors participated in drafting this manuscript and approved of the final version. The authors acknowledge Dr. Rosy Abou-Joudé, Department of pathology, Hotel-Dieu de France, Beyrouth, Lebanon, for her help in generating the cytology images used in Figure 1.

\section{REFERENCES}

[1] Jensen RT, Cadiot G, Brandi ML, de Herder WW, Kaltsas G, Komminoth P, et al. ENETS Consensus Guidelines for the management of patients with digestive neuroendocrine neoplasms: functional pancreatic endocrine tumor syndromes. Neuroendocrinology. 2012;95(2):98-119.

[2] Ciaravino V, Robertis RD, Martini PT, Cardobi $\mathrm{N}$, Cingarlini S, Amodio A, et al. Imaging presentation of pancreatic neuroendocrine neoplasms. Insights Imaging. 2018 Oct 9;1-11.

[3] Caplin ME, Pavel M, Ćwikła JB, Phan AT, Raderer M, Sedláčková E, et al. Lanreotide in metastatic enteropancreatic neuroendocrine tumors. N Engl J Med. 2014 Jul 17;371(3):22433.

[4] Wolin EM. The Expanding Role of Somatostatin Analogs in the Management of Neuroendocrine Tumors. Gastrointest Cancer Res GCR. 2012;5(5):161-8.

[5] Singhi AD, Klimstra DS. Well-differentiated pancreatic neuroendocrine tumours (PanNETs) and poorly differentiated pancreatic neuroendocrine carcinomas (PanNECs): concepts, issues and a practical diagnostic approach to high-grade (G3) cases. Histopathology. 2018 Jan;72(1):168-77.

[6] Oronsky B, Ma PC, Morgensztern D, Carter CA. Nothing But NET: A Review of Neuroendocrine Tumors and Carcinomas. Neoplasia N Y N. 2017 Dec;19(12):991-1002.

[7] Auernhammer CJ, Spitzweg C, Angele MK, Boeck S, Grossman A, Nölting S, et al. Advanced neuroendocrine tumours of the small intestine and pancreas: clinical developments, controversies, and future strategies. Lancet Diabetes Endocrinol. 2018;6(5):404-15.

[8] Scoazec J-Y, Couvelard A. Classification des tumeurs neuroendocrines pancréatiques: nouveautés introduites par la classification OMS 2017 des tumeurs des organes endocrines et perspectives. Ann Pathol. 2017 Dec 1;37(6):444-56.

[9] Duan K, Mete O. Algorithmic approach to neuroendocrine tumors in targeted biopsies: Practical applications of immunohistochemical markers. Cancer Cytopathol. 2016 Dec 1;124(12):871-84.

[10] Deguelte S, de Mestier L, Hentic O, Cros J, Lebtahi R, Hammel P, et al. Preoperative imaging and pathologic classification for pancreatic neuroendocrine tumors. J Visc Surg. 2018 Apr 1;155(2):117-25.

[11] Rinke A, Müller H-H, Schade-Brittinger C, Klose K-J, Barth P, Wied M, et al. Placebocontrolled, double-blind, prospective, randomized study on the effect of octreotide LAR in the control of tumor growth in patients 
with metastatic neuroendocrine midgut tumors: a report from the PROMID Study Group. J Clin Oncol Off J Am Soc Clin Oncol. 2009 Oct 1;27(28):4656-63.

[12] Blesl A, Krones E, Pollheimer MJ, Haybaeck J, Wiesspeiner U, Lipp RW, et al. Downgrading of a G3 Neuroendocrine Tumor to a G2 Tumor: Can First-Line Cytotoxic Chemotherapy Change the Tumor Biology? Case Rep Oncol. 2017 Dec 20;10(3):1121-6.

[13] Kwekkeboom DJ, Kam BL, Essen M van, Teunissen JJM, Eijck CHJ van, Valkema R, et al. Somatostatin receptor-based imaging and therapy of gastroenteropancreatic neuroendocrine tumors. Endocr Relat Cancer. 2010 Mar 1;17(1):R53-73.

[14] Davi' MV, Cosaro E, Piacentini S, Reimondo G, Albiger N, Arnaldi G, et al. Prognostic factors in ectopic Cushing's syndrome due to neuroendocrine tumors: a multicenter study. Eur J Endocrinol. 2017 Apr;176(4):451-9.
[15] Herrera-Martínez AD, Pedraza-Arevalo S, LLópez F, Gahete MD, Gálvez-Moreno MA, Castaño JP, et al. Type 2 Diabetes in Neuroendocrine Tumors: Are Biguanides and Statins Part of the Solution? J Clin Endocrinol Metab. 2019 Jan 1;104(1):57-73.

[16] Feelders RA, Newell-Price J, Pivonello R, Nieman LK, Hofland LJ, Lacroix A. Advances in the medical treatment of Cushing's syndrome. Lancet Diabetes Endocrinol. 2018 Jul 19.

[17] Pivonello R, Ferone D, Lamberts SWJ, Colao A. Cabergoline plus lanreotide for ectopic Cushing's syndrome. N Engl J Med. 2005 Jun 9;352(23):2457-8.

[18] Bruno OD, Danilowicz K, Manavela M, Mana D, Rossi MA. Long-term management with octreotide or cabergoline in ectopic corticotropin hypersecretion: case report and literature review. Endocr Pract Off J Am Coll Endocrinol Am Assoc Clin Endocrinol. 2010 Oct;16(5):829-34.

Citation: Nada Younes, Anne-Sophie Azzi, Bassam Abboud, Chawki Atallah. Can Somatostatin Receptor Analogs Re-Differentiate Pancreatic Neuroendocrine Tumors: A Case Report. ARC Journal of Clinical Case Reports. 2020; 6(4):9-13. DOI: https://doi.org/10.20431/2455-9806.0604003.

Copyright: (C) 2020 Authors. This is an open-access article distributed under the terms of the Creative Commons Attribution License, which permits unrestricted use, distribution, and reproduction in any medium, provided the original author and source are credited. 